\title{
22986
}

\section{Patients' perspectives on exposure to environmental contaminants: a missing piece in pulmonary rehabilitation?}

Air pollution, Education, COPD - management

\author{
S. Souto-Miranda ${ }^{1}$, S. Melo-Dias ${ }^{1}$, C. Valente ${ }^{2}$, C. Freitas ${ }^{3}$, A. Margarida Sousa ${ }^{4}$, A. Catarina
} Sousa $^{5}$, A. Marques ${ }^{1}$

${ }^{1}$ Lab3R - Respiratory Research and Rehabilitation Laboratory, School of Health Sciences, University of Aveiro, Aveiro Portugal; iBiMED - Institute of Biomedicine, School of Health Sciences, University of Aveiro, Aveiro, Portugal - Aveiro (Portugal), ${ }^{2}$ Department of Pulmonology, Centro Hospitalar do Baixo Vouga, E.P.E, Aveiro, Portugal - Aveiro (Portugal), ${ }^{3}$ Lab3R - Respiratory Research and Rehabilitation Laboratory, School of Health Sciences, University of Aveiro, Aveiro Portugal - Aveiro (Portugal), ${ }^{4}$ iBimED - Institute of Biomedicine, School of Health Sciences, University of Aveiro, Aveiro, Portugal - Aveiro (Portugal), ${ }^{5} \mathrm{CICECO}$ Aveiro Institute of Materials, University of Aveiro, Aveiro, Portugal - Aveiro (Portugal)

Chronic obstructive pulmonary disease (COPD) is influenced by environmental factors, such as indoor/outdoor contaminants. However, patients' views on environmental exposure are little-known and this is an often-neglected topic in psychoeducational sessions in pulmonary rehabilitation (PR). We explored the views of patients with COPD about indoor/outdoor environment and its influence on their health and the impact of an environmental educational session within PR, on their daily living.

Focus groups were conducted before and after community-based PR. An educational session was designed and included in PR after the first focus group. Data were analysed with thematic analysis.

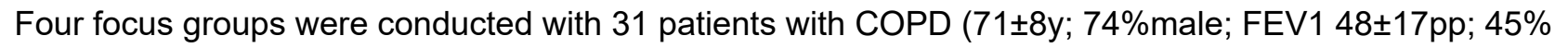
GOLD2; $58 \%$ GOLDB). Prior to PR, 3 themes emerged: 1) "just seeing the tip of the iceberg" since some awareness existed but a feeling of probably not knowing the real impact was shared; 2) "feeling resigned" where patients acknowledged having poor strategies to avoid contaminants and felt there was little they could do; and 3) "willingness to learn" as they reported the need for an educational session to improve their knowledge on environmental exposures. After PR, 2 themes were found: 1) "gratefulness" where patients shared that the session was helpful for themselves and significant others; and 2) "personal change", where patients expressed how their behaviour changed towards more health-enhancing strategies to deal with contaminants.

Environmental education within PR is valued by patients and leads to specific behaviour changes to deal with contaminants' exposure. Further attention to this topic is warranted. 